\title{
Normal-incidence efficiencies of 4800 -grooves/mm-ruled replica gratings with multilayer and gold coatings in the 125-325-Å wavelength region
}

\author{
John F. Seely, Tetsuya Watanabe, Tatsuo Harada, Jack C. Rife, and William R. Hunter
}

\begin{abstract}
The normal-incidence efficiencies of two 4800-grooves/mm ruled replica gratings, one with a dualbandpass molybdenum/silicon multilayer coating and the other with a gold coating, were measured by use of synchrotron radiation in the 125-325-Å wavelength region. The peak reflectance of the multilayer coating was $22 \%$ in the first Bragg order near $235 \AA$ and $28 \%$ in the second Bragg order near $126 \AA$. The peak efficiency of the multilayer grating was $2.6 \%$ in the first diffraction order near $225 \AA$ and $0.3 \%$ in the second diffraction order near $125 \AA$. The efficiencies of the multilayer grating were much higher than the corresponding efficiencies of the gold grating. The characterization of the surfaces of the gratings by atomic force microscopy indicated $\mathrm{rms}$ microroughness values in the $5-18-\mu \mathrm{m}^{-1}$ frequency range of $12-20 \AA$ for the multilayer grating and 22-32 $\AA$ for the gold grating. Both gratings had bumpy surface features larger than the nominal groove height. The rather large surface roughness and groove irregularities had a detrimental effect on the grating efficiencies. (C) 1999 Optical Society of America

OCIS codes: $\quad 050.0050,050.1950,340.7470,310.6860$.
\end{abstract}

\section{Introduction}

High-dispersion diffraction gratings are required for the observation of spectral line profiles and Doppler shifts in solar, astrophysical, and laboratory radiation sources. A 4800-grooves/mm grating has been considered for the planned Solar-B satellite mission to study the dynamics of the Sun's corona, photosphere, and transition regions. ${ }^{1,2}$ The grating operates at normal incidence and has a multilayer coating to enhance the efficiency in an extreme ultraviolet (XUV) spectral region of spectroscopic diagnostic interest. Multilayer-coated diffraction gratings with groove densities as high as 3600 grooves $/ \mathrm{mm}$ have been experimentally characterized using synchrotron radiation in the XUV and soft x-ray spectral regions..$^{3,4}$

In this paper we report the characterization of a

J. F. Seely (john.seely@nrl.navy.mil) and J. C. Rife are with the Naval Research Laboratory, Washington, D.C. 20375. T. Watanabe is with the National Astronomical Observatory, Mitaka, Tokyo 181, Japan. T. Harada is with the Tokyo Metropolitan University, Hachioji, Tokyo 192-03, Japan. W. R. Hunter is with SFA, Incorporated, 1401 McCormick Drive, Landover, Maryland 20785.

Received 6 October 1998; revised manuscript received 4 January 1999.

0003-6935/99/101920-06\$15.00/0

(C) 1999 Optical Society of America 4800-grooves/mm ruled replica grating with a $\mathrm{Mo} / \mathrm{Si}$ multilayer coating. The multilayer coating was designed to have high normal-incidence reflectance in two bandpasses corresponding to the first Bragg order at a wavelength of $235 \AA$ and the second Bragg order near $126 \AA$. The normal-incidence grating efficiency was measured using synchrotron radiation and was compared with the efficiency of a gold-coated grating that was replicated from the same master grating. This research demonstrates for the first time to our knowledge the enhancement of the normal-incidence efficiency of a 4800 -grooves $/ \mathrm{mm}$ grating in the XUV region by using a multilayer coating.

\section{Atomic Force Microscopy}

Two replicas of a flat, ruled master grating were produced by Hitachi Ltd. The master grating had 4800 grooves $/ \mathrm{mm}$ and a nominal blaze angle of $3.7^{\circ}$. The master grating was an initial test ruling for this groove frequency and blaze angle.

Each replica grating had a grooved area of $40 \mathrm{~mm}$ by $40 \mathrm{~mm}$ and an aluminum surface that resulted from the replication process. A gold coating was deposited on one replica grating and a multilayer coating on the other.

The surfaces of the gold and multilayer gratings were characterized using a Topometrix Explorer Scanning Probe Microscope, a type of atomic force 


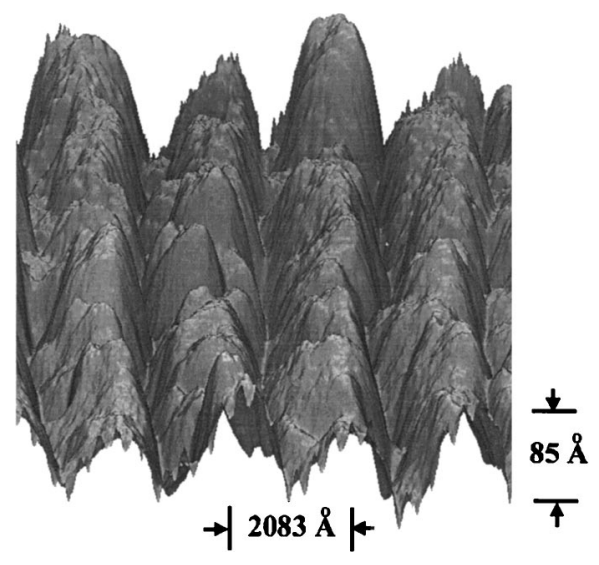

Fig. 1. AFM image of the multilayer grating. The vertical scale was expanded to show the texture of the groove pattern.

microscope (AFM). The AFM images typically had $500 \times 500$ pixels and a scan range of $1-20 \mu \mathrm{m}$ (pixel size 20-400 ̊). The silicon probe had a pyramid shape. The base of the pyramid was $3-6 \mu \mathrm{m}$ in size, the height of the pyramid was 10-20 $\mu \mathrm{m}$, and the height-to-base ratio was approximately 3. The tip of the pyramid had a radius of curvature less than 200 $\AA$. The AFM scans were performed using the noncontact resonating mode, where the change in the oscillation amplitude of the probe is sensed by the instrument.

A surface topology reference sample was used to optimize the AFM scanning parameters, to calibrate the height scaling of the instrument, and to evaluate the performance of the AFM. The surface topology reference sample consisted of an array of approximately square holes fabricated on the silicon dioxide surface of a silicon die by VLSI Standards Inc. The top surface of the die was coated with a thin layer of platinum. The hole array had a pitch of $3 \mu \mathrm{m}$ and a hole depth of $180 \AA$.

An AFM image of four grooves of the multilayer grating is shown in Fig. 1 . The scan was performed across the grooves over a range of $1 \mu \mathrm{m}$ (20-Å pixels). The vertical scale in Fig. 1 was expanded to reveal the texture of the grating surface. The grooves are rather irregular in shape and have rounded peaks. The multilayer grating, as well as the gold grating, has bumpy surface features that are larger than the nominal groove height.

The groove height was measured in AFM images recorded at several different places near the center of the multilayer grating. The average groove height was $90 \AA$, and the standard deviation was $25 \AA$. The facet angles were determined by measuring the angles from the horizontal of the grooves's faces. In Fig. 1, the average angles of the left-facing and rightfacing facets were $3.8^{\circ}$ and $5.2^{\circ}$, respectively. Similar values were obtained for the gold grating.

The rms microroughness was calculated from an area under the power spectral density curves that were derived from the AFM images. The microroughness derived from different scans over the sur- faces of the gratings had considerable variation. The rms microroughness values in the $5-18-\mu m^{-1}$ frequency range (within the grooves) were $12-20 \AA$ for the multilayer grating and 22-32 A for the gold grating. This implies that the gold grating was significantly rougher than the multilayer grating.

Similar results were derived from the comparison of the microroughness of three ruled replica gratings with 3600 grooves $/ \mathrm{mm}^{3}$ One replica grating had a multilayer coating, another a gold coating, and the third was uncoated and had an aluminum surface resulting from the replication process. Each of the three gratings was replicated from the same master grating. The microroughness of the uncoated and the multilayer-coated gratings were comparable, and the microroughness of the gold grating was higher. Gold coatings typically have a microroughness up to $40 \AA$, whereas Mo/Si multilayer coatings on smooth substrates have an effective microroughness of approximately $10 \AA .^{5}$ As discussed in Section 3 , the effective microroughness is based on computer modeling of the reflectance of the multilayer coating and results from the intrinsic microroughness of the layer interfaces and the interdiffusion of the layer materials.

\section{Reflectance of the Multilayer Coating}

The same multilayer coating was deposited on one replica grating substrate and two mirror substrates. The mirror substrates were 2.5 in. $(6.4 \mathrm{~cm})$ in diameter and had a concave radius of curvature of $2.5 \mathrm{~m}$. The mirrors were superpolished with a microroughness of $1 \AA$. The flat grating and the two concave mirrors were designed for a Czerny-Turner spectrometer optical system with resolving power of $>30,000$ that will be described in a future publication. The multilayer coatings were deposited by OSMIC Inc. using the sputtering technique.

The reflectances of the multilayer mirrors, as well as the grating efficiencies, were measured at the $\mathrm{Na}$ val Research Laboratory beamline X24C at the National Synchrotron Light Source at Brookhaven National Laboratory. The synchrotron radiation was dispersed by a monochromator that had a resolving power of 600.6,7 Thin filters suppressed the radiation from the monochromator in the higher harmonics. The wavelength scale was established by the geometry of the monochromator and the absorption edges of the filters. The accuracy of the wavelength scale was $0.1-0.2 \%$ of the incident wavelength. The incident radiation was approximately $90 \%$ polarized with the electric field vector in the plane of incidence ( $p$ polarization).

The measured reflectances of the two mirrors, designated Mirror 1 and Mirror 2, in the 124-134-A and 175-325- $\AA$ wavelength regions are shown in Figs. 2(a) and 2(b). In the longer wavelength region, the reflectance was measured at angles of incidence of $7^{\circ}$ and $10^{\circ}$. The peak reflectance of $22 \%$ occurred near a wavelength of $235 \AA$, and this corresponds to the first Bragg order of the multilayer coating. In the shorter wavelength region, the reflectance was measured at an angle of incidence of $7^{\circ}$. The peak re- 

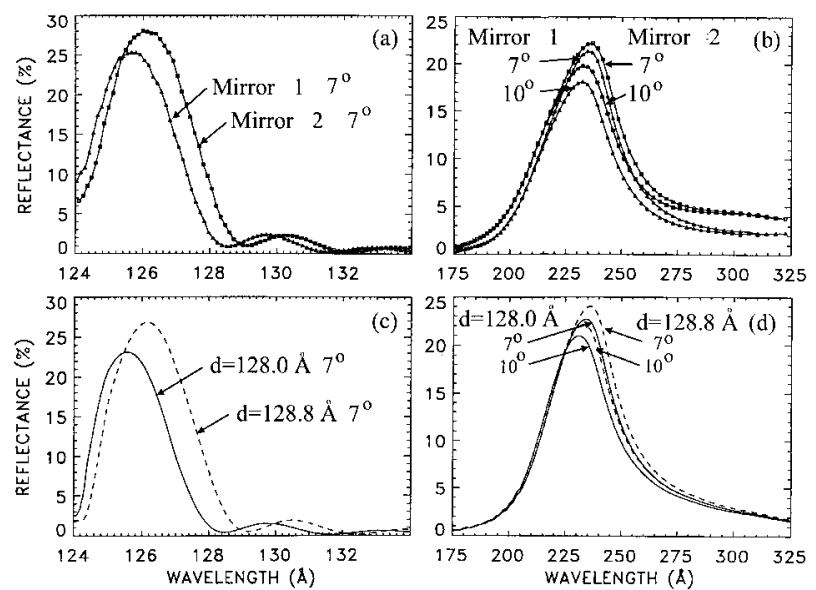

Fig. 2. Measured reflectances of the multilayer-coated Mirror 1 and Mirror 2 in (a) the second Bragg order and (b) the first Bragg order wavelength regions. The calculated reflectances of the two mirrors in (c) the second Bragg order and (d) the first Bragg order wavelength regions. The angles of incidence are indicated.

flectance of $28 \%$ occurred near $126 \AA$, and this corresponds to the second Bragg order.

The calculated reflectances are shown in Figs. 2(c) and 2(d). The reflectance was calculated using a computational model composed of $18 \mathrm{Mo} / \mathrm{Si}$ periods (36 layers total). The complex amplitude reflectance was calculated at each material interface, and a Debye-Waller factor was applied at each interface to simulate microroughness and interdiffusion. The Mo and Si densities were 10.2 and 2.33 grooves $/ \mathrm{cm}^{3}$, respectively. The optical constants were derived from the scattering factors of Ref. 8. The period thickness was adjusted slightly from the expected value, based on the deposition parameters, so that the wavelength of the peak reflectance agreed with the observed wavelength. The inferred period thicknesses for Mirror 1 and Mirror 2 were 128.0 and 128.8 $\AA$, respectively. The Debye-Waller microroughness parameters were adjusted so that the values of the peak calculated and measured reflectances were in agreement. The inferred Debye-Waller parameters for Mirror 1 and Mirror 2 were 11 and $10 \AA$, respectively.

As can be seen in Fig. 2, the calculated and measured reflectances are in good overall agreement. The shift to shorter wavelength with increasing angle of incidence, for a multilayer coating with a fixed period thickness, is consistent with the Bragg condition. The slightly different period thicknesses for Mirrors 1 and 2, 128.0 and $128.8 \AA$, may result from small variations in the deposition parameters or from slightly different positions relative to the sputtering sources.

\section{Measured Grating Efficiency}

The grating efficiency measurements were performed in the same reflectometer as the reflectance measurements. The gratings were mounted with the grooves perpendicular to the plane of incidence. In
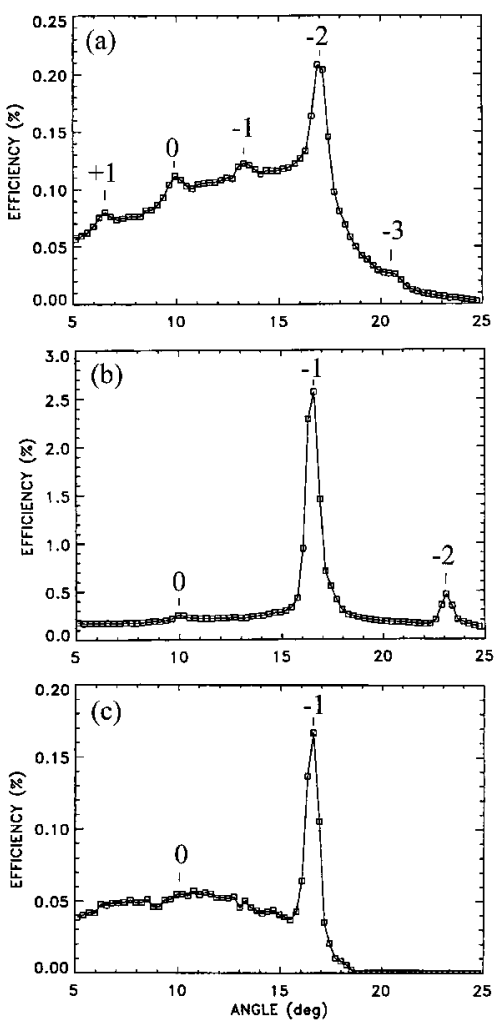

Fig. 3. Efficiency of the multilayer grating measured at an angle of incidence of $10^{\circ}$ and wavelengths of (a) $125 \AA$ and (b) $225 \AA$. (c) The efficiency of the gold grating measured at an angle of incidence of $10^{\circ}$ and a wavelength of $225 \AA$. The inside $(m>0)$ and outside $(m<0)$ diffraction orders are identified.

this orientation, the electric field vector of the incident radiation was perpendicular to the grooves. The groove facets with the smaller angle $\left(3.8^{\circ}\right)$ faced the incident radiation. The detector was scanned in angle about the grating for fixed values of the incident wavelength. The accuracy of the detector and sample angles was $0.1^{\circ}$. The efficiency was calculated by taking the ratio of the intensity of the radiation diffracted by the grating and the intensity of the incident radiation.

The measured efficiencies of the multilayer grating at an angle of incidence of $10^{\circ}$ (measured from the normal to the surface of the grating) and at wavelengths of 125 and $225 \AA$ are shown in Figs. 3(a) and $3(\mathrm{~b})$. The inside $(m>0)$ and outside $(m<0)$ diffraction orders are identified. Measurements at diffraction angles less than $5^{\circ}$ were not possible because of occultation of the direct beam by the detector. As expected by the blaze condition, the -2 order is strongest at the shorter wavelength and the -1 order is strongest at the longer wavelength. The efficiency of the gold grating at $10^{\circ}$ incidence and a wavelength of $225 \AA$ is shown in Fig. 3(c). The efficiency of the gold grating in the 125-A wavelength region was too low to measure.

The efficiencies of the multilayer and gold gratings were measured at an angle of incidence of $10^{\circ}$ and at a number of fixed wavelengths in the $175-335$-A wave- 

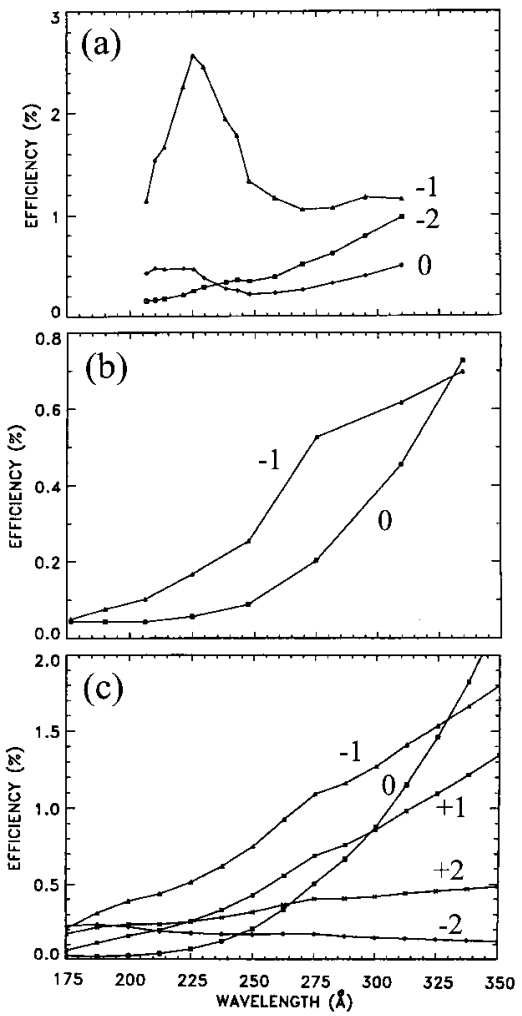

Fig. 4. Peak efficiencies of (a) the multilayer grating and (b) the gold grating measured at an angle of incidence of $10^{\circ}$. (c) The calculated efficiency of the gold grating at an angle of incidence of $10^{\circ}$. The diffraction orders are indicated.

length range. The peak efficiencies in the observed orders were determined. The peak efficiencies, measured as functions of wavelength, of the multilayer and gold gratings are shown in Figs. 4(a) and 4(b). The calculated efficiencies of the gold grating are shown in Fig. 4(c) and are discussed in Section 5.

By comparing the measured efficiencies in Figs. 4(a) and 4(b), it is apparent that the efficiency of the multilayer grating is much higher than the efficiency of the gold grating, particularly in the wavelength region where the multilayer coating has high reflectance. The peak efficiency of the multilayer grating in the -1 diffraction order is $2.6 \%$ at a wavelength of $225 \AA$, whereas the efficiency of the gold grating is $0.16 \%$ at the same wavelength, a factor of 16 smaller.

As can be seen in Fig. 2(b), the multilayer coatings on Mirrors 1 and 2 have peak reflectance at a wavelength of $233 \AA$ for an angle of incidence of $10^{\circ}$. The -1 order of the multilayer grating has peak efficiency at a wavelength of $225 \AA$ for an angle of incidence of $10^{\circ}$ as shown in Fig. 4(a). Because of the $3.8^{\circ}$ nominal facet angle, the average angle of incidence on the facets is $13.8^{\circ}$, and this slightly larger angle (compared with $10^{\circ}$ ) is consistent with the shift in peak wavelength from 233 to $225 \AA$. Similar peak wavelength shifts have been observed for other blazed multilayer gratings. ${ }^{3}$ Based on previous experience with the deposition process, the wavelength shift is too large to result from differences in the period

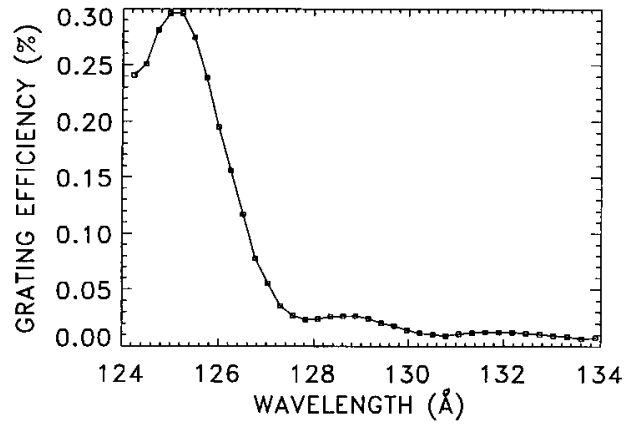

Fig. 5. Measured efficiency of the multilayer grating in the -2 diffraction order and at an angle of incidence of $6^{\circ}$.

thicknesses of the multilayer coatings on the mirrors and the grating.

The detector was positioned on the -2 diffraction order of the multilayer grating, and the wavelength was scanned over the 124-134- $\AA$ wavelength range. The -2 efficiency is shown in Fig. 5 for the case of an angle of incidence of $6^{\circ}$. For larger angles of incidence, the peak efficiency moved below the silicon $L$ absorption edge at a wavelength of $124.2 \AA$ and was not observable.

The measured efficiency of the multilayer grating in the -2 order and at an angle of incidence of $6^{\circ}$ peaks at a wavelength of $125.0 \AA$ as shown in Fig. 5 . The measured reflectance of the multilayer coatings on Mirrors 1 and 2, in the second Bragg order and at an angle of incidence of $7^{\circ}$, peak at wavelengths of 125.8 and 126.2 $\AA$ as shown in Fig. 2(a). The shorter peak wavelength for the multilayer grating efficiency is consistent with the larger effective angle of incidence on the facets. That is, the sum $\left(9.8^{\circ}\right)$ of the grating angle of incidence $\left(6^{\circ}\right)$ and the blaze angle $\left(3.8^{\circ}\right)$ is larger than the mirror angle of incidence $\left(7^{\circ}\right)$.

The groove efficiency of the multilayer grating was inferred by taking the ratio of the measured efficiency and the reflectance of the multilayer coating. The reflectance of the multilayer coating on the grating was calculated using the same computational model as described in Section 3, except that the DebyeWaller microroughness parameter was set equal to $16 \AA$. This value was based on the average microroughness (12-20 $\AA$ ) determined from the AFM images. The calculated multilayer reflectances were $7.1 \%$ and $14 \%$ at wavelengths of 125 and $225 \AA$, respectively. The rather large microroughness of $16 \AA$ has a significant detrimental effect on the multilayer reflectance, particularly in the shorter wavelength region. Using the peak grating efficiencies of $0.3 \%$ in the -2 order at a wavelength of $125 \AA$ and $2.6 \%$ in the -1 order at $225 \AA$, the corresponding inferred groove efficiencies are $4 \%$ and $19 \%$. These groove efficiencies are comparable to those of other ruled replica blazed gratings in these wavelength regions. ${ }^{3}$ In general, the relatively low groove efficiencies of ruled replica multilayer gratings, as compared with gratings fabricated by the holographic ion-etched blazed technique, are attributed to the high micro- 
roughness and irregular groove profile of the ruled replica gratings. ${ }^{3}$

\section{Calculated Grating Efficiency}

The efficiency of the gold grating was calculated using an improved version of the computer program developed by Goray and Chernov. ${ }^{9,10}$ The program is based on the modified integral method and accounts for the finite conductivity of the gold coating, the nonideal groove profile as determined by the AFM images, and the polarization of the incident radiation. The program was used in previous research to model the efficiency of 3600 -grooves/mm and 2400 grooves/mm blazed gratings. ${ }^{4,11}$

The efficiency was calculated for a representative groove profile, having 103 data points, that was selected from the AFM image shown in Fig. 1. In the calculation, the grating was orientated as for the measurements, with the $3.8^{\circ}$ facet facing the incident radiation. The angle of incidence, measured from the normal to the grating surface, was $10^{\circ}$. The grooves were perpendicular to the plane of incidence, and the electric field vector was in the plane of incidence (and perpendicular to the grooves). The efficiencies of the $0, \pm 1, \pm 2, \pm 3, \pm 4$, and \pm 5 diffraction orders were calculated. The calculated efficiencies for the $0, \pm 1$, and \pm 2 orders are shown in Fig. 4(c). The efficiencies in the \pm 4 and \pm 5 orders were much smaller than in the \pm 1 and \pm 2 orders.

By comparing the calculated and measured efficiencies of the gold grating in the 0 and -1 orders, as shown in Figs. 4(b) and 4(c), it is apparent that the calculated efficiencies are higher than measured. This could result from the bumpy features appearing in the AFM image of Fig. 1 that are higher than the nominal groove height. These surface features, which have a spatial scale comparable to or larger than the groove spacing, are not accounted for in the calculation that uses a single nominal groove profile. However, the relative calculated values of the efficiencies in the 0 and -1 orders, including the crossing at a wavelength of $330 \AA$, are in good agreement with the measured relative efficiencies.

In Figs. 4(b) and 4(c), the slight enhancement of the efficiency of the gold grating near $275 \AA$ results from the blaze effect. The grating's blaze wavelength, ${ }^{5}$ for an angle of incidence of $10^{\circ}$ and a $3.8^{\circ}$ facet angle, is $268 \AA$. Ideally, the wavelength of peak reflectance of the multilayer coating would match the blaze wavelength. For the present experiment, it was decided to design the dual-bandpass multilayer coating so that the peak reflectance in the second Bragg order occurred at a wavelength just above the silicon $L$ absorption edge at $124.2 \AA$ (see Fig. 2). In this case, the silicon spacer material has high transmittance, and the reflectance in the second Bragg order is optimal. However, twice the period of the multilayer coating (256 $\AA$ ) was smaller than the blaze wavelength $(268 \AA)$, and thus there was a small mismatch between the multilayer coating and the blazed grating substrate. The blaze effect is typically much broader in wavelength than the multilayer reflec- tance profile, so a small mismatch is usually not detrimental to the efficiency of the multilayer grating. ${ }^{5}$

\section{Conclusions}

The normal-incidence efficiency of a 4800-grooves/ mm grating with a multilayer coating was characterized in the XUV wavelength region. The efficiency of the multilayer grating was enhanced by the relatively high reflectance of the multilayer coating in the wavelength regions corresponding to the first and second Bragg orders of the multilayer coating. The efficiencies in these wavelength regions were much higher than the efficiencies of a gold-coated grating replicated from the same master grating. The peak groove efficiencies of $19 \%$ and $4 \%$ at wavelengths of 225 and $125 \AA$ are comparable to those of ruled replica gratings with 3600 grooves $/ \mathrm{mm}$. The efficiencies are considerably smaller than the efficiencies of multilayer gratings fabricated by the holographic ionetched blazed technique, and this is attributed to the rather large microroughness and groove profile irregularities of the ruled replica gratings. The 4800grooves $/ \mathrm{mm}$ master grating was an initial test ruling, and subsequent 4800 -grooves/mm ruled gratings are expected to have improved microroughness and groove profile regularity.

This research was supported by NASA project W19193. Part of the research was done at the National Synchrotron Light Source, which is sponsored by the U.S. Department of Energy under contract DEAC02-76CH00016. The two replica gratings were provided by Hitachi Ltd., Instrument Division, Katsuta, Ibaraki 312, Japan. The AFM images were recorded in collaboration with Kaj Stolt of Analytical Answers Inc.

\section{References}

1. T. Watanabe, H. Hara, and T. Harada, "Spectroscopic observations in Solar-B," in UV and X-Ray Spectroscopy of Astrophysical and Laboratory Plasmas, K. Yamashita and T. Watanabe, eds. (Universal Academy Press, Tokyo, 1996), pp. 219-250.

2. T. Harada, H. Sakuma, K. Takahashi, T. Watanabe, H. Hara, and T. Kita, "Design of a high-resolution extreme ultraviolet imaging spectrometer with aberration-corrected concave gratings," Appl. Opt. 37, 6803-6810 (1998).

3. J. F. Seely, M. P. Kowalski, W. R. Hunter, T. W. Barbee, Jr., R. G. Cruddace, and J. C. Rife, "Normal-incidence efficiencies in the 115-340- $\AA$ wavelength region of replicas of the Skylab 3600-line/mm grating with multilayer and gold coatings," Appl. Opt. 34, 6453-6458 (1995).

4. W. R. Hunter, J. F. Seely, M. P. Kowalski, J. C. Rife, and T. W. Barbee, Jr., "Grazing-incidence efficiencies in the 28-42- $\AA$ wavelength region of replicas of the Skylab 3600-line/mm concave grating with multilayer and gold coatings," Appl. Opt. 36, 6411-6415 (1997).

5. J. F. Seely, M. P. Kowalski, W. R. Hunter, J. C. Rife, T. W. Barbee, Jr., G. E. Holland, C. N. Boyer, and C. M. Brown, "On-blaze operation of a Mo/Si multilayer-coated, concave diffraction grating in the $136-142-\AA$ wavelength region and near normal incidence," Appl. Opt. 32, 4890-4896 (1993).

6. J. C. Rife, H. R. Sadeghi, and W. R. Hunter, "Upgrades and recent performance of the grating/crystal monochromator," Rev. Sci. Instrum. 60, 2064-2067 (1989). 
7. W. R. Hunter and J. C. Rife, "An ultrahigh vacuum reflectometer/goniometer for use with synchrotron radiation," Nucl. Instrum. Methods Phys. Res. A 246, 465-468 (1986).

8. B. L. Henke, E. M. Gullikson, and J. C. Davis, "X-ray interactions: photoabsorption, scattering, transmission, and reflection at $\mathrm{E}=50-30,000 \mathrm{eV}, \mathrm{Z}=1-92$," At. Data Nucl. Data Tables 54, 181-342 (1993). Updated optical constants were obtained from the internet site cindy.lbl.gov/optical_constants.

9. L. I. Goray, "Numerical analysis for relief gratings working in the soft $\mathrm{x}$-ray and XUV region by the integral equation meth- od," in X-Ray and UV Detectors, R. B. Hoover and M. W. Tate, eds., Proc. SPIE 2278, 168-172 (1994).

10. L. I. Goray and B. C. Chernov, "Comparison of rigorous methods for x-ray and XUV grating diffraction analysis," in X-Ray and Extreme Ultraviolet Optics, R. B. Hoover and A. B. C. Walker, eds., Proc. SPIE 2515, 240-245 (1995).

11. M. P. Kowalski, J. F. Seely, L. I. Goray, W. R. Hunter, and J. C. Rife, "Comparison of the calculated and the measured efficiencies of a normal-incidence grating in the 125-225- $\AA$ wavelength region," Appl. Opt. 36, 8939-8943 (1997). 